\title{
AN ELEMENTARY PROOF OF A THEOREM CONCERNING INFINITELY CONNECTED DOMAINS
}

\author{
R. J. SIBNER ${ }^{1}$
}

\begin{abstract}
Using classical complex function theory, it is shown that any infinitely connected plane domain is conformally equivalent to a domain whose isolated boundary components are analytic Jordan curves. This allows an elementary proof to be given of the result that a domain with countably many boundary components is conformally equivalent to a domain bounded by analytic Jordan curves.
\end{abstract}

1. It is an immediate consequence of the Riemann mapping theorem that any domain of finite connectivity can be mapped conformally onto a domain whose boundary components are analytic Jordan curves. In recent work ([2], [3]) the author has shown that this result holds for infinitely connected domains (with countably many boundary components). The proof, by transfinite induction, uses only the standard theory of normal families and the following: every plane domain is conformally equivalent to a domain whose isolated boundary components are analytic Jordan curves. (Points are to be considered as degenerate analytic Jordan curves, but in any case if they are isolated they can be ignored.) The proofs given in [2] and [3] of this statement use deep results in the theory of quasiconformal mappings. We give here a direct "normal family proof" (a simplified version of the transfinite induction argument in [3]). Once this is done, the entire proof of the general theorem (see §3) becomes elementary-using only classical function theory (cf. [3, Note, p. 418]).

2. We recall the classical proof for finitely connected domains. If the boundary components of such a domain $D_{0}$ are indexed by $k=1, \cdots, n$ and this indexing is preserved under conformal maps of the domain, then one obtains maps $f_{k}(0 \leqq k \leqq n)$ of $D_{0}$ onto domains $D_{k}$ whose first $k$ boundary components are analytic Jordan curves. This is done by successively applying the Riemann mapping theorem to the domain bounded

Received by the editors March 31, 1972.

AMS (MOS) subject classifications (1970). Primary 30A 30.

Key words and phrases. Conformal mapping, infinitely connected domains, analytic Jordan curves.

${ }^{1}$ Research partially supported by NSF Grant GP 7952X3. 
by the $k$ th boundary component of $D_{k-1}$. This can always be done leaving invariant two fixed points. If $h_{k-1}^{*}$ is this map and $h_{k-1}$ the restriction of $h_{k-1}^{*}$ to $D_{k-1}$ we let $f_{k}=h_{k-1} \circ f_{k-1}$ and $D_{k}=f_{k}\left(D_{0}\right)$. The first $k-1$ boundary components of $D_{k}$ are analytic Jordan curves because $h_{k-1}$ is the restriction of a map which is conformal in a neighborhood of these components. The $k$ th boundary component of $D_{k}$ is the unit circle.

These remarks can be applied to an infinitely connected domain $D_{0}$ whose (nondegenerate) isolated boundary components (of which there are at most countably many) are indexed by the natural numbers. With the above notations let, for $1<j<\infty, h_{i j}^{*}=h_{j-1}^{*} \circ \cdots \circ h_{i}^{*}$ so that $h_{i j}^{*}$ is conformal in the domain bounded by those boundary components of $D_{i}$ indexed by $i+1, \cdots, j$ and let $h_{i j}: D_{i} \rightarrow D_{j}$ be the restriction of $h_{i j}^{*}$ to $D_{i}$. We obtain then the

LEMMA. Let $D_{0}$ be an arbitrary domain. Then there exists a collection of domains $D_{i}(i=1,2, \cdots)$, maps $f_{i}: D_{0} \rightarrow D_{i}$ and $($ for $i<j)$ maps $h_{i j}: D_{i} \rightarrow D_{j}$ satisfying:

(1) The first $i$ boundary components of $D_{i}$ are analytic Jordan curves.

(2) $h_{i j}$ is the restriction to $D_{i}$ of a map $h_{i j}^{*}$ of the domain bounded by the boundary components of $D_{i}$ indexed by $i+1, \cdots, j$.

(3) $h_{i j}=h_{k j} \circ h_{i k}(i<k<j)$.

(4) $f_{j}=h_{i j} \circ f_{i}$.

(A formal induction proof could easily be given.)

We state the

PROPOSITION [3]. An arbitrary plane domain is conformally equivalent to a domain whose isolated boundary components are analytic Jordan curves.

Proof. The maps $h_{i j}^{*}$ are univalent in the domain $D_{i}^{*}$ bounded by all the boundary components of $D_{i}$ except those indexed by $1, \cdots, i$. Consequently, they form a normal family (the distortion theorem implies that they are locally uniformly bounded-see e.g. [1]). A diagonalization argument shows that there is a subsequence $j_{n}$ such that $h_{i j_{n}}^{*}$ converges (uniformly on compact subsets of $D_{i}^{*}$ ) for each $i$ to a map $h_{i \infty}^{*}$ which is univalent in $D_{i}^{*}$. (It is not a constant because of the normalization on $h_{k}^{*}$.) If $h_{i \infty}$ is the restriction of $h_{i \infty}^{*}$ to $D_{i}$ then, for $i<k<\infty, h_{i \infty}=h_{k \infty} \circ h_{i k}$ and, by (4), $f_{j_{n}} \rightarrow f_{\infty}: D_{0} \rightarrow D_{\infty}$ where $f_{\infty}=h_{i \infty} \circ f_{i}$ for every $i$.

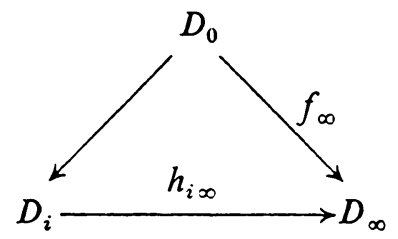


For $i>l$ the $l$ th boundary component of $D_{i}=f_{i}\left(D_{0}\right)$ is an analytic Jordan curve and $h_{i \infty}$ is the restriction of a map conformal in a neighborhood of the first $i$ boundary components of $D_{i}$ (in particular, in a neighborhood of the $l$ th). Hence the $l$ th boundary component of $D_{\infty}$ is an analytic Jordan curve for arbitrary $l$ and the proposition is proved.

Note. The stronger result that any domain is conformally equivalent to a domain whose isolated boundary components are circles was obtained in [2] with the use of quasiconformal mappings. It also can be obtained as above, using the result for finitely connected domains and the reflection principle.

3. For completeness, we recall briefly how the above proposition is used in the proof of the general theorem: a domain with countably many boundary components is conformally equivalent to a domain bounded by analytic Jordan curves [3].

The collection $\Gamma_{1}$ of boundary components is provided with a natural metric space structure. One considers the chain $\Gamma_{1} \supset \Gamma_{2} \supset \cdots \supset \Gamma_{\alpha} \supset$ $\Gamma_{\alpha+1} \supset \cdots$ indexed by the ordinal numbers where if the ordinal $\alpha$ has a predecessor $\alpha-1$ then $\Gamma_{\alpha}$ is the derived set of $\Gamma_{\alpha-1}$ and if $\alpha$ is a limit ordinal then $\Gamma_{\alpha}=\bigcap \Gamma_{\beta}, \beta<\alpha$.

Next, one considers an induction hypothesis analogous to (1)-(4) above. Here again, it is the richness of the hypotheses which is the key to the method of proof.

The step to an ordinal $\alpha$ with a predecessor is made by applying the above proposition to the domain bounded by $\Gamma_{\alpha-1}$, observing that the isolated boundary components of this domain are $\Gamma_{\alpha-1}-\Gamma_{\alpha}$.

For a limit ordinal, one uses a normal family argument, similar to that used in the proof of the proposition of $\$ 2$, but now on a sequence of ordinals tending to the limit ordinal.

\section{REFERENCES}

1. Z. Nehari, Conformal mapping, McGraw-Hill, New York, 1952. MR 13, 640.

2. R. J. Sibner, Domains bounded by analytic Jordan curves, Bull. Amer. Math. Soc. 76 (1970), 61-63. MR 41 \#445.

3. - 'Uniformizations' of infinitely connected domains, Advances in the Theory of Riemann Surfaces, Ann. of Math. Studies, no. 66, Princeton Univ. Press, Princeton, N.J., 1971, pp. 407-420.

Institute for Advanced Study, Princeton, New Jersey 08540

Current address: Department of Mathematics, Polytechnic Institute of Brooklyn, Brooklyn, New York 11201 